AsClepIO. Revista de Historia de la Medicina y de la Ciencia

69 (2), julio-diciembre 2017, p190

ISSN-L:0210-4466

http://dx.doi.org/10.3989/asclepio.2017.13

DOSSIER: ESCENARIOS DE LAS PRÁCTICAS PSIQUIÁTRICAS EN AMÉRICA LATINA (SIGLOS XIX Y XX) /

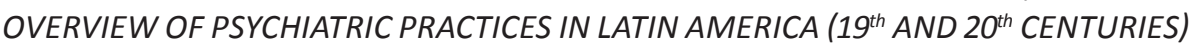

\title{
EL HOSPICIO NACIONAL DE ALIENADOS EN LA PRENSA DE RÍO DE JANEIRO (1903-1911)
}

\author{
Ana Teresa A. Venancio \\ Fundación Oswaldo Cruz, Río de Janeiro, Brasil \\ anavenancio4@gmail.com \\ ORCID iD: http://orcid.org/0000-0001-7827-9927 \\ José Roberto Saiol \\ Fundación Oswaldo Cruz, Río de Janeiro, Brasil \\ joseroberto_hist@hotmail.com \\ ORCID iD: http://orcid.org/0000-0002-2361-9282
}

Recibido: 07 abril 2017; Aceptado: 03 junio 2017.

Cómo citar este artículo/Citation: Venancio, Ana Teresa A. y Saiol, José Roberto (2017), "El Hospicio Nacional de Alienados en la prensa de Río de Janeiro (1903-1911)", Asclepio 69 (2): p190. doi: http://dx.doi.org/10.3989/asclepio.2017.13

RESUMEN: Este trabajo analiza la forma en que la prensa de la capital republicana difundió informaciones durante la primera década del siglo XX, contribuyendo a la formación de opiniones acerca del Hospicio Nacional de Alienados (HNA), primera institución psiquiátrica brasileña que abrió sus puertas en la ciudad de Río de Janeiro en 1852. Para esta investigación hemos analizado las noticias sobre el Hospicio en dos diarios: O Paiz y Correio da Manhã. Hemos seguido en ella tanto la historiografía brasileña más reciente, como la referente a la historia de la psiquiatría en Iberoamérica, con objeto de relativizar la relevancia otorgada a la psiquiatría y al manicomio en su función de control social, así como de apuntar su carácter híbrido y polifacético en las noticias que se publicaron en la prensa de la capital federal. En los dos periódicos, el conjunto variado de menciones sobre el HNA pone en juego tres puntos de vista: los dos primeros se centran en la descripción y el retrato de la vida institucional, llevado a cabo por prensa, en sus diferentes aspectos (administrativo, asistencial, científico); mientras que el tercero analiza aquellas situaciones en que el Hospicio se contempla como una solución para acontecimientos que perturbaban la vida de la ciudad.

PALABRAS CLAVE: Hospicio Nacional de Alienados; Manicomio; Psiquiatría; Historia; Brasil.

\section{THE NATIONAL ASYLUM FOR THE INSANE IN THE RIO DE JANEIRO PRESS (1903-1911)}

ABSTRACT: During the first decade of the twentieth century, the press of the republican capital disseminated representations and helped to form opinions about the National Hospice of Alienated Persons (HNA), the first Brazilian psychiatric institution, which began to function in the city of Rio de Janeiro in 1852. For this investigation we have analyzed the news about the hospice in two newspapers - O Paiz and Correio da Manhã. We follow here the most recent Brazilian historiography, as well as the history of psychiatry in Ibero-American countries, to discuss the emphasis given to the social control function of the asylum and to point out its hybrid and multifaceted character in the news that circulated in the press of the federal capital. In the two newspapers, the varied set of references to the HNA puts into circulation three points of view: the first two express how the institutional life, in its different aspects (administrative, welfare, scientific) is described and portrayed by the press; while the third point of view brings together the situations in which the hospice is considered as a solution for events that disturb the life of the city.

KEY WORDS: Hospicio Nacional de Alienados; Asylum; Psychiatry; History; Brazil.

Copyright: @ 2017 CSIC. Este es un artículo de acceso abierto distribuido bajo los términos de la licencia Creative Commons Attribution (CC BY) España 3.0. 


\section{PRESENTACIÓN}

Trataremos de analizar aquí la forma en que la prensa de la capital republicana difundió informaciones durante la primera década del siglo XX, contribuyendo así a la formación de opiniones acerca del Hospicio Nacional de Alienados (HNA) ${ }^{1}$, primera institución psiquiátrica brasileña, fundada en 1841, durante el segundo periodo imperial (1840-1189), si bien solo comenzaría a funcionar en 1852, bajo la administración de la Santa Casa de la Misericordia, y que permaneció abierta hasta la década de 1940. La historiografía de la psiquiatría en Brasil ha apuntado el papel central del Hospicio Nacional de Alienados (HNA) en la configuración de un lugar institucional, simbólico y social para la locura desde el período del Imperio, al atribuir su fundación a los anhelos de un grupo de médicos, reunidos en torno a la Sociedad de Medicina y Cirugía de Río de Janeiro, quienes desde 1830 exigían medidas para atender a los locos dispersos por las calles de la Corte; sin olvidar tampoco al poder imperial, dado que su fundación surgió en homenaje a la coronación de D. Pedro II, segundo emperador de Brasil, en $1841^{2}$. (Machado et al., 1978; Engel, [1995] 2001; Teixeira, 1998; Gonçalves y Edler, 2009).

Sin embargo, tal y como demuestran Gonçalves y Edler (2009) y Ribeiro (2015), en dicha historiografía se observan divergencias en cuanto a dos aspectos: el tipo de población acogida en el Hospicio y el modo en que se producía la admisión de los enfermos. Atendiendo a las series originales de fuentes, las interpretaciones más recientes acerca del Hospicio de Pedro II en el período imperial han rescatado nuevas evidencias sobre la existencia, ya en esa época, de una preocupación y de una práctica médica dirigidas a los alienados, en contraposición con la visión que lo consideraba como mero lugar de exclusión social. Aunque la segregación fuera finalmente el nefasto resultado del manicomio, reducir las dinámicas sociales que constituyen la historia de la institución a la etiqueta de la "exclusión" significaría minimizar la importancia y la complejidad de los procesos que allí se gestaron y desarrollaron. Tales procesos también se deben entender como conformadores de la especialidad médica y de la asistencia a la alienación mental.

El estudio más detallado de las diversas fuentes relativas a la admisión de alienados pone en cuestión "la percepción de que el Hospicio se había creado para apartar de la circulación a los individuos de conducta desviada que vagaban por las calles de la ciudad" (Gonçalves y Edler, 2009, p.406). Nuevos documentos prueban la existencia de flujos de admisión de dife- rentes grupos durante el período imperial, como, por ejemplo, el gran contingente de militares del Ejército y de la Marina procedentes de la Guerra del Paraguay (1864-1870), internados en las décadas de 1860 y 1870 (Gonçalves y Edler, 2009, p.402; Ribeiro, 2016). Además, estos estudios constatan que, en la admisión de los internos, los médicos tenían un papel relevante (Gonçalves y Edler, 2009, pp. 404-405).

La lucha por la aplicación combinada del tratamiento moral (preconizado por Pinel y por Esquirol), junto con la terapéutica con medicamentos (recomendada por médicos contemporáneos a estos), evidencia la actualización de los criterios científico-técnicos puestos en marcha por los médicos [...] (Gonçalves, 2013, p. 75).

Esta historiografía más reciente sobre el Hospicio de Pedro II sigue la línea de la historia de la psiquiatría en Iberoamérica, al relativizar la afirmación sobre el papel central de control social desempeñado por la psiquiatría y los manicomios, defendido por los estudios de Foucault o inspirados en él. Las críticas dirigidas a estos trabajos se centran tanto en la necesidad de análisis de las especificidades históricas nacionales y locales y de investigación de cuestiones diversas sobre las relaciones saber-poder, como en la importancia del trabajo minucioso con las fuentes (Campos y Huertas, 2008; Huertas, 2009, p.20 y 2012; Sacristán, 2005, 2009, p.176). Tales estudios han demostrado la complejidad de la constitución y el desarrollo de la psiquiatría y de los manicomios, productos y productores de discursos y prácticas ligados a la generación de conocimientos científicos y a la profesionalización de una especialidad médica; determinados en su conjunto por preocupaciones humanitarias, a la par que atentos a un control social considerado necesario. Aparte de eso, se cuestiona el propio papel del Estado como organizador del control social ejercido por las instituciones psiquiátricas, como apuntan las investigaciones sobre el Hospital Nacional de Alienadas de Buenos Aires (Ablard, 2008, p.5) y el Manicomio General La Castañeda en los alrededores de la ciudad de México (Sacristán, 2005, p.25; Ríos Molina, 2009, pp. 26-27).

Desde esta perspectiva, cada vez son más frecuentes en la historiografía iberoamericana los trabajos que destacan el carácter social híbrido y polifacético del manicomio, a partir del análisis de su vida cotidiana y de las diversas fuentes, al establecer un diálogo entre las distintas voces y producir así una historia del "manicomio con el sujeto” (Sacristán, 2009, p. 167 y 178). El manicomio se percibe como un lugar de internación de grupos heterogéneos a lo largo del tiempo, según demuestra Ríos Molina (2009, pp.50-51), en el que 
se cruzan discursos y prácticas que no son unívocas, generadas por actores sociales diversos, aunque igualmente importantes en el proceso de definición sobre quién debía ser internado en el Hospicio: la dirección de la institución, los médicos, los funcionarios, los pacientes y sus familiares, tal y como revelan Ablard (2008, p. 127 ss) y Ríos Molina (2009, pp. 27-32).

A partir de este enfoque de la historia social y cultural, nos preguntamos sobre los discursos y las prácticas relativas al Hospicio que sobrepasaron los muros de la institución y que se propagaron por la sociedad en general, gracias a la divulgación que de ellas hicieron las publicaciones periódicas, en concreto, los diarios $^{3}$. Las noticias en la prensa daban visibilidad a la institución ante el público lego, contribuyendo a la formación de una opinión sobre lo que debía ser considerado como locura y cómo debía abordarse. Pretendemos en este artículo, por tanto, presentar la complejidad de la identidad del Hospicio Nacional de Alienados durante el inicio de la Primera República, demostrando la coexistencia de distintas representaciones que, en relación con él, circularon en la prensa de la capital.

Para esta investigación hemos analizado las noticias sobre el Hospicio en dos periódicos: O Paiz y Correio da Manhã ${ }^{4}$. Como sabemos, en el inicio de la República brasileña, no existía radiodifusión regular para la población, sino que era la prensa el medio más destacado de comunicación y de difusión de diversos tipos de informaciones. Los periódicos constituían, por consiguiente, un vehículo formador de opiniones, así como de divulgación de comportamientos, actitudes y acciones políticas (Martins y Luca, 2006, p. 11). Durante el período estudiado, y sobre todo a partir de los últimos años de la década de 1890, con la llegada a la presidencia de civiles, se abre la fase de la "prensa con fueros de industria" (Martins y Luca, 2006, p.37). Esta "prensa profesionalizada (1889-1930)" (Martins y Luca, 2006), se caracterizaba, a grandes rasgos, por la sustitución del negocio artesanal por el industrial, y por la remuneración a los periodistas por los asuntos que publicaban. Los periódicos aquí analizados han sido elegidos en su calidad de representantes de esta prensa profesional, en contraste con el resto que se caracterizaba por su precariedad de medios (Tavares, 1980 , p. 30). Se trata de periódicos consolidados y financieramente independientes, impresos en la ciudad de Río de Janeiro, de edición diaria, aunque también con diferentes posicionamientos respecto al contexto político republicano, lo que permite la comparación y el cotejo de las fuentes ${ }^{5}$.
Analizaremos aquí el período republicano de la historia del Hospicio, iniciado bajo la gestión de Juliano Moreira como director del Hospicio Nacional de Alienados (HNA) a partir de 1903. Este es el momento en que se adoptan una serie de medidas renovadoras en relación con la asistencia a los alienados, como la primera gran reforma republicana del Hospicio, que se produce con la publicación de los decretos $n$. 응 1.132/1903 y n.o 5.125/1904, los cuales instituían y reglamentaban una ley de reorganización de la asistencia en el país. En este contexto, Juliano Moreira se erige como líder del proceso, no solo en lo tocante a la implantación de una moderna asistencia, sino también en la institucionalización de la ciencia psiquiátrica (Venancio e Carvalhal, 2005). Cabe recordar que, poco antes de la designación de Juliano Moreira para la dirección del HNA, esta institución aparecía citada constantemente en los periódicos de la época por la situación extrema en que se encontraba: asistencia precaria, convivencia de internos menores y adultos, malversación de recursos financieros por el administrador, quien incluso llegó a ser arrestado ${ }^{6}$.

El marco final de nuestro análisis se sitúa en 1911, cuando Juliano Moreira asume además la dirección general de la Asistencia a Alienados de toda la capital de la República (Muñoz et al. 2011, p. 93), con el desafío de planificar y poner en marcha la asistencia general a los alienados, más allá de la prestada por el Hospicio. Ese mismo año se inauguró la Colonia de Alienadas en Engenho de Dentro, a la que fueron trasladadas internas de la Sección Esquirol del HNA (Facchinetti et al. 2010, p.735). También se promulga el decreto n.o 8.834 de 11 de julio de 1911, que -reorganiza la asistencia a alienados- y cambia el nombre del Hospicio por el de Hospital Nacional de Alienados. Gracias a ese cuadro asistencial más amplio, se producirán a partir de entonces nuevas reformulaciones en la institución (Muñoz et al., 2011, pp. 94-95).

\section{DEL IMPERIO A LA REPÚBLICA: LA PRENSA EN LA CIUDAD DE RÍO DE JANEIRO}

La prensa de la capital de la República a principios del siglo XX apenas contaba con un siglo de vida, dado que había llegado al país con la familia real portuguesa en 1808 (Nascimento y Zanlorenzi, 2006, p. 38). Con Portugal a punto de ser invadido por las tropas de Napoleón Bonaparte y sin condiciones militares para hacerles frente, la corte portuguesa de D. João VI decidió trasladarse a Brasil y establecerse en la ciudad de Río de Janeiro. Este hecho supondría, para la ciudad colonial, un "cambio de fisionomía", pues, "entre 
otros aspectos, se esbozó entonces una vida cultural, con el acceso a los libros y la existencia de cierta circulación de las ideas" (Fausto, 2002, p. 69). En la segunda mitad del siglo XIX, cuando se inauguró el Hospicio, la prensa de la ciudad de Río de Janeiro era marcadamente literaria en detrimento del periodismo político (Sodré, 1983, p. 192; Nascimento y Zanlorenzi, 2006, p. 49). La prensa imperante era, o bien académica, sobre todo en las facultades de derecho, o bien literaria, dirigida a los jóvenes y estudiantes, incluyendo al joven público femenino, a quien dedicaba artículos sobre el matrimonio (Sodré, 1983, pp. 196-198).

A partir de la década de 1870, se propagaron nuevas ideas entre la élite y en la prensa, en especial las referentes a la cuestión de la esclavitud, cuya defensa o rechazo polarizaba el debate y ponía en evidencia intereses (Sodré, 1983, p. 223; Alonso, 2002). El fin de la esclavitud en 1888 desencadenó una transferencia explícita de apoyo al movimiento republicano por parte de los grandes propietarios de haciendas de café que, en torno a militares disidentes de la Guardia Nacional, intensificaron los ataques contra el Imperio, hasta la proclamación de la República en 1889.

En ese contexto, vio la luz O Paiz en 1884, que se destacó por su participación en las campañas abolicionista y republicana durante los últimos años de la monarquía. El primer redactor jefe del periódico fue Rui Barbosa, pronto sustituido por Quintino Bocaiúva (1836-1912), quien, nacido en Itaguaí (Río de Janeiro), se mudó en 1850 a São Paulo. A su vuelta a Río de Janeiro, trabajó en el Spectador Brasileiro y después fue redactor-jefe del Diário do Rio de Janeiro (Sodré, 1983, p. 195). Este último, relanzado en 1860 gracias a su labor, era uno de los diarios más populares de la ciudad y se caracterizaba por su acción combativa, así como por su excelente divulgación del panorama literario (Sodré, 1983, p. 191). En 1867, Bocaiúva viaja a los Estados Unidos, pasando por Argentina y Paraguay. De regreso a la capital del Imperio, colabora en la redacción del Manifesto Republicano, publicado el 3 de diciembre de 1870 en el primer número de $A$ República (Sodré, 1983, p. 212). Entre 1874 y 1883, "restauró O Globo" y, a continuación, fundó O Paiz (Sodré, 1983, p. 195).

Tras la proclamación de la República, se inicia el periodo de los presidentes militares, con los mariscales Deodoro da Fonseca (1889-1891) y Floriano Peixoto (1891-1894). Ya en este inicio, se imponía el reto de estructurar una nueva organización política. Según Souza (1968), la primera Constitución Brasileira de 1891 lo resumiría bien:
Federalismo, presidencialismo y ampliación del régimen representativo son las tres grandes coordenadas legales de la Primera República [...], unidas a las características de una estructura económica definida por la gran propiedad [...] y su desarrollo como colonia exportadora de materias primas (Souza, 1968, p. 186).

La importancia de los estados en la organización de esa nueva estructura política, junto con la participación de un contingente mayor de electores, que hasta entonces había permanecido al margen, provocaron manifestaciones de descontento con el "principio federativo", como ocurriera con la Revolución Federalista de 1892, en el estado de Rio Grande do Sul.

Con el paso del Imperio a la Republica, la producción cafetera de los estados del centro-sur del país mantuvo su preponderancia, si bien no de modo uniforme. A pesar de continuar "determinada por el latifundio y dirigida al mercado externo", asumió una nueva configuración a finales del siglo XIX, cuando el país dominaba "dos tercios del mercado mundial del café" y "la producción agrícola [esclavista] comenzara a basarse en el brazo asalariado" (Souza, 1968, p. 187). De este modo, el poder de los propietarios de café mantenía su capacidad de presión ante el gobierno central. Por otra parte, "organizado en base al trabajo asalariado, que permitía un mejor uso de los medios de producción ya existentes en el país, la estructura cafetera propició el desarrollo de un núcleo urbano industrial", estimulando así el desarrollo de un mercado interno (Souza, 1968, p. 188).

El primer presidente elegido por elección directa, Prudente de Morais (1894-1898), representará preferencialmente los intereses de los cafeteros en detrimento de los intereses de los militares. Al comienzo de su gobierno abandona, poco a poco, las medidas adoptadas por Floriano Peixoto. Sin embargo, dos años después de su investidura, ha de apartarse del cargo por motivos de salud, asumiendo la presidencia su segundo Manuel Vitoriono, defensor de las ideas de Floriano. Los periódicos, por su parte, publicaban viñetas cómicas y chistes que criticaban cáusticamente a los presidentes (Lustosa 1989a, 1989b). El presidente Prudente de Morais aparecía como "Prudente de Más" en la visión del famoso periodista e ilustrador Angelo Agostini, por el hecho de atender los intereses de los simpatizantes de Floriano (Lustosa, 1989a, p. 17-18). La dimisión del presidente también fue objeto de la sátira cuando, desde el humor, se conectaron sus problemas de salud "con la grave crisis nacional del momento" (Lustosa, 1989a, p. 19) 
En 1898, en plena inestabilidad económica, herencia de Prudente de Morais, asumió la presidencia Campos Sales (1841-1913). Jurista y republicano histórico, era uno de tantos políticos formados en la "Facultad de Derecho de São Paulo para ocupar los grandes cargos públicos de la llamada 'República de los bachilleres' " (Lustosa, 1989a, p. 30). De familia con credo latitudinario, así como exseñores de esclavos, fue víctima de chistes y apodos relativos a su origen social y a su vanidad (Lustosa, 1989a, p. 29).

Desde el punto de vista económico, Campos Sales trató de resolver el problema de la deuda externa con la firma de un acuerdo con los bancos acreedores ingleses, conocido como funding loan, consistente en la suspensión por varios años del pago de la deuda y de sus intereses. En el intento de combatir la inflación, puso en marcha una dura política económica para los ciudadanos, por la cual se interrumpió la emisión de papel moneda, se recortaron de gastos, se crearon nuevos impuestos y se aumentaron los existentes. No obstante, el liberalismo económico adoptado en la Primera República se mantuvo asociado al poder de las oligarquías, configurándose como un liberalismo oligárquico (Resende, 2008, p.91).

Desde el punto de vista político, Campos Sales instituyó la "política de los estados" o "política de los gobernadores", con la que se ganó el apoyo del Congreso al establecer relaciones de acercamiento político entre el gobierno central (el presidente de la República), los estados y sus gobernadores y los municipios, bajo el control de las viejas oligarquías de terratenientes (Lustosa, 1989a, p.30). La "política del café con leche", que se desplegó a partir de entonces, representaría bien ese proceso: la sucesión de presidentes y vicepresidentes oriundos de los respectivos estados cafeteros y productores de leche-São Paulo y Minas Gerais- se iniciaba con Campos Sales, quien gobernaría hasta 1902, y con sus posteriores sucesores, el presidente paulista Rodrigues Alves y su vicepresidente, el mineiro Afonso Pena (1902-1906), quien sería presidente entre 1906 y $1909 .^{7}$

Rodrigues Alves, amigo personal de Campos Salles, fue elegido por significativa mayoría de los votos directos. Su principal adversario en este proceso fue Quintino Bocaiúva ${ }^{8}$ que, aunque desde 1901 ya no dirigía o Paiz, mantenía su influencia sobre la línea editorial del diario. Los hitos del gobierno de Rodrigues Alves por su trascendencia histórica fueron: el Convenio de Taubaté (1906), que instituía una política de revalorización del café por la que el gobierno pasaba a comprar y almacenar el excedente de la producción, con vistas a mantener alto el valor del producto; la política liderada por Oswaldo Cruz como director general de Salud Pública (1903-1909), marcada por las revueltas populares, así como por la de la Escuela Militar de 1904, conocida como Revuelta de la Vacuna; y la reforma de la ciudad de Río de Janeiro, según los planes del alcalde e ingeniero Pereira Pasos (1902-1906).

En ese contexto, el Correio da Manhã salía a la calle por primera vez. Creado en 1901 por el abogado Edmundo Bittencourt (1866-1943), desde su fundación fue un periódico de opinión y de oposición (Carvalho, 1980, p. 99). De acuerdo con Sodré,

el Correio da Manhã surgía para romper, efectivamente, la letanía de loores al gobierno de Campos Sales que presidía la política de estancamiento, terriblemente onerosa para las clases populares. Pretendía romper la placidez aparente alcanzada a través del soborno y de la corrupción sistemática por la compra institucionalizada de la opinión de la prensa (Sodré, 1983, p. 287).

Así, el diario emprendió diversas campañas -como la contraria a la obligatoriedad de la vacunación y al autoritarismo gubernamental en las medidas de saneamiento de la ciudad de Río de Janeiro- saliendo siempre en defensa de las causas de interés popular, como fue "el caso del hígado podrido":

En una carnicería de la Rua do Rosário, propiedad de uno de los socios del Matadero de Santa Cruz, el diario compró un trozo de hígado de buey que estaba en mal estado y lo dejó expuesto en la conserjería. Pidiendo a Oswaldo Cruz que lo examinara, este se negó, alegando que se trataba de una cuestión política. El hígado se llevó al laboratorio de la Policía Militar, que confirmó la irregularidad. Al sostener tales luchas, el diario consolida la confianza y el apoyo de una cartera de clientes urbana que le garantizaba fuerza y prestigio (Carvalho, 1980, p. 100).

O Paiz, por su parte, se había mantenido políticamente cercano al Gobierno y se caracterizaba por un "extremo servilismo" durante la joven República (Sodré, 1983, p. 278). Aunque defendiera de forma significativa la campaña republicana, su actuación estaba profundamente marcada por un "situacionismo" bajo continua sospecha, y por una fuerte identificación con la estructura política de la Primera República (1889-1930). ${ }^{9}$

\section{EL HOSPICIO NACIONAL DE ALIENADOS EN LA CIUDAD: NOTICIAS EN LAS PÁGINAS DE $O$ PAIZ Y CORREIO DA MANHÃ (1903-1911)}

Desde al menos la segunda mitad del siglo XIX, Río de Janeiro vivió transformaciones que incorporaron 
nuevos espacios al entorno urbano -con vistas a establecer la separación de usos y clases sociales en la ciudad-, impulsadas por la introducción de medios de transporte, como tranvías para el área central y trenes para barrios más periféricos (Abreu, 2013, pp. 36-39 y 43). Ciertas partes del centro de la ciudad serían objeto de reformas urbanísticas -pavimentación, instalación de iluminación a gas, así como implantación de servicios de alcantarillado en 1862-, aunque, paradójicamente, esta zona continuó concentrando las viviendas, por lo común colectivas e insalubres, de la población más pobre que, sin condiciones de movilidad, ocupaba el centro de la ciudad a la busca cotidiana de oportunidades de trabajo (Abreu, 2013, p.42). Tras la proclamación de la República, se observa también "la difusión de la ideología que asociaba el estilo de vida 'moderno' con la localización residencial a la orilla del mar" (Abreu, 2013, p. 47). Todos estos factores trajeron cambios a la ciudad, culminados con la reforma de Pereira Pasos, que instituía un nuevo trazado urbanístico, alejando así de sus áreas centrales a la población más pobre.

En este contexto, también se fue transformando el espacio donde estaba situado el Hospicio Nacional de Alienados, la Praia da Saudade, aunque más len- tamente que otras zonas de la ciudad. El Hospicio se ubicaba en la antigua Chácara do Vigário Geral, en un área de distintas fincas y propiedades, como la de Manuel Antunes Suzano ${ }^{10}$, quien también poseía otras tierras en la zona. Muy cerca de la Praia da Saudade se encontraba la Praia Vermelha, donde había un antiguo fuerte desde principios del siglo XVIII. En la segunda mitad del XIX, la Praia Vermelha se convirtió en sede de la Escuela Militar que, en 1860, construye en el antiguo fuerte una edificación bordeando la playa, entre los cerros de la Urca y de Babilônia. En 1872 se inaugura al lado del manicomio otra institución asistencial: el Imperial Instituto de Niños Ciegos.

El aislamiento y el difícil acceso a la zona comenzaron a superarse a principios del siglo XX. Con el compromiso de la compañía de tranvías de ampliar sus líneas hasta Praia Vermelha, entre 1900 y 1903 (Abreu, 2013, p. 48), se construyeron allí varios pabellones en 1908, con ocasión de la Exposición Conmemorativa del primer centenario de la apertura de los puertos de Brasil. El Pabellón de las Industrias se instaló en la edificación de la Escuela Militar, ya que esta había sido trasladada a Realengo, barrio alejado del centro de la ciudad, tras la Revuelta de la Vacuna (Pereira, 2010, p.17). De acuerdo con esta autora, la exposición

Figura 1. Mapa de la región de la Praia da Saudade

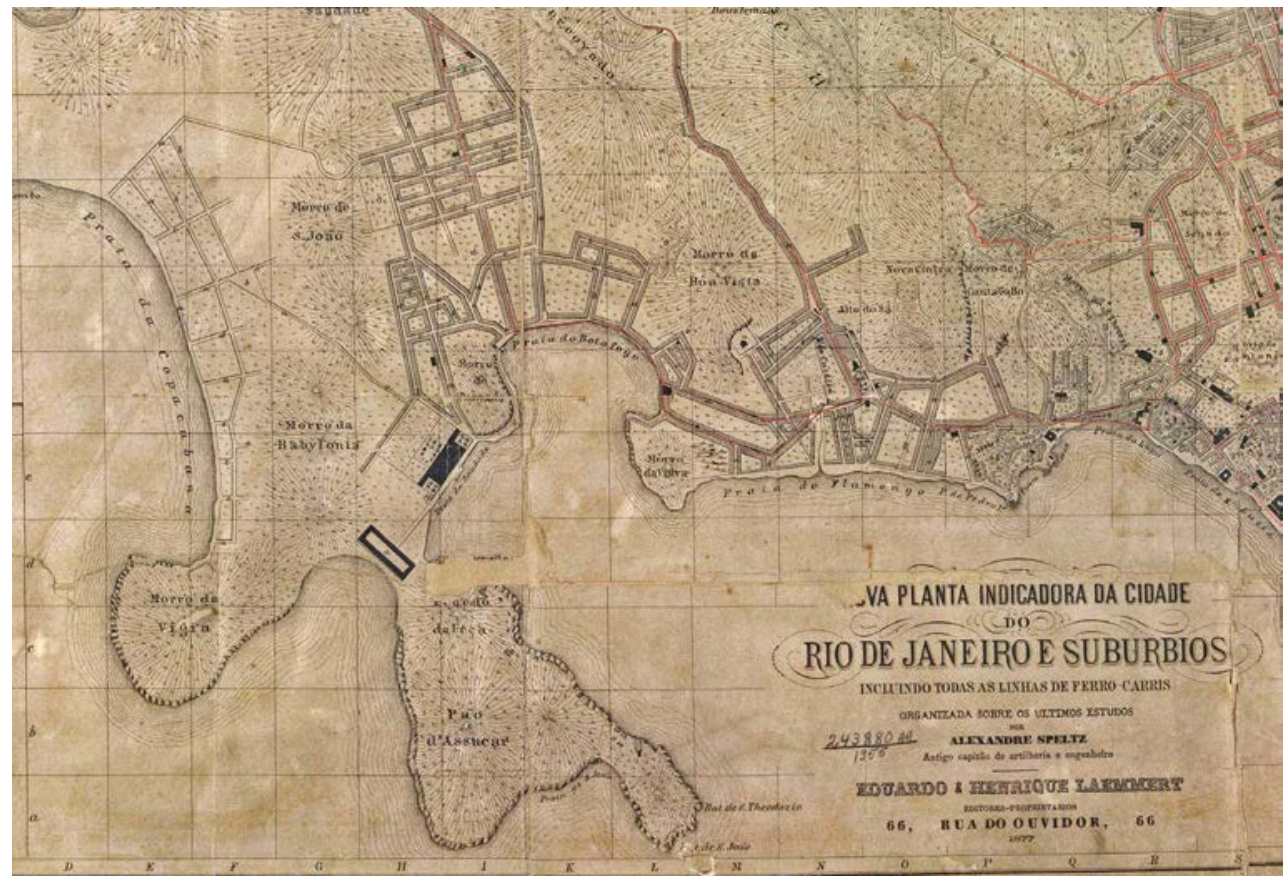

El edificio rectangular de líneas gruesas es la Escuela Militar. El edificio rectangular en negro, situado en frente a la Praia da Saudade, es el Hospicio Nacional de Alienados. Speltz, Alexander (1877), Planta indicadora de la ciudad de Río de Janeiro y sus suburbios, incluidas todas as líneas de ferrocarril, Rio de Janeiro, Eduardo e Henrique Laemmert. 
de 1908 servía de escaparate para la República, al divulgar la modernidad de las máquinas, las inversiones del capital y el progreso de la ciencia del país. Se instalaron pabellones sobre la actuación de los estados y contó con la participación de varias instituciones, como el Hospicio de Alienados, el Observatorio Astronómico, la Dirección General de Estadística, la Academia Nacional de Medicina, el Instituto Histórico y Geográfico Brasileño, entre otras (Pereira, 2010, pp. 24-25). Después de la exposición, el emplazamiento donde se hallaba el Hospicio se volvió más popular y concurrido, sobre todo por bañistas e interesados en las competiciones de remo que empezaron a celebrarse en aquella zona -específicamente en la Praia de Botafogo-, donde la administración de Pereira Passos promovió este tipo de eventos que contaban con gran asistencia de público de diversas clases sociales (Melo, 2001, pp.96-97). ${ }^{11}$

Mientras que en 1902 O Paiz y Correio da Manhã informaban ampliamente del problema del desfalco en las arcas del Hospicio Nacional de Alienados y de la comisión creada para depurar los hechos (Azevedo, 2012), a partir del año siguiente, las noticias destacaban preferentemente los cambios en la institución ${ }^{12}$, proyectados o ya en marcha. La presencia del Hospicio Nacional de Alienados (HNA) en las páginas de $O$ Paiz y Correio da Manhã aumenta entre los años 1903 y 1911. La denominación de la institución en las páginas de esos diarios responde al nombre oficial de la institución a lo largo del período: desde 1903 la referencia a la institución republicana como"nacional" -Hospicio Nacional- es mucho más frecuente que la denominación Hospicio de Alienados; esta última se utiliza cada vez menos, aunque no desaparezca por completo. El nombre Hospital Nacional, instituido por ley en 1911, casi no aparece, a no ser puntualmente en 1905, y solo vuelve a emplearse en las noticias a partir de 1909 en O Paiz, y a partir de 1910 en el Correio da Manhã.

Muchas veces el nombre del Hospicio se menciona en estos diarios como parte de la lista de instituciones objeto de actos del poder público; en otros casos, se trata de meras vicisitudes en la carrera de un funcionario, relativas a sus vacaciones, cese o nombramiento; o acerca de un paciente fallecido, noticia que se cubría en la columna de "fallecimientos" de Correio da Manhã. Esas breves referencias institucionales también se recogen en las notas sociales, cuando se hace referencia a médicos del Hospicio, siendo el más citado su director, Juliano Moreira. Menciones puntuales a otros médicos se hallan también en los anuncios de los diarios, como el del "consultorio del Dr. Cardinal, médico del Hospicio"13.

En ambos diarios, la mayor parte de las alusiones al Hospicio eran pequeños textos, notas sin autoría, siendo mucho menos frecuentes los artículos extensos y firmados. Las notas que divulgaban los actos públicos $^{14}$-nombramientos, ceses, licencias, concursos, rendición de cuentas, autorizaciones para obras- se publicaban a menudo en $O$ Paiz, bajo los títulos de "Pagos del Tribunal de Cuentas", "Cámara" y "Ecos y Hechos". Otras columnas en ese diario que recogían notas sobre el HNA fueron "La Semana", "Sección Policial", "Vida Forense" y "Vida Social". En el Correio da Manhã, las pequeñas notas sobre el Hospicio se encuentran en las columnas "Ayer" y "Hoy", cuando se trataba de eventos sociales o acontecimientos en la ciudad. Otras columnas del periódico informaban acerca de la ciudad: "Caídas y derrames" -normalmente pequeños textos satíricos- y "En la policía y en las calles", en la que se daban a conocer noticias policiales, entre ellas, la mayoría de los sucesos en los que alguno de los implicados acababa por ser recluido en el Hospicio Nacional de Alienados. También la columna "Ejército" informaba sobre el movimiento de los internos militares en esa institución.

En el período analizado, las imágenes sobre el HNA son escasas en O Paiz y Correio da Manhã, si se compara con los años siguientes de la década de 1910. 0 Paiz sólo había publicado una noticia, el 22 de enero de 1901, en la que aparecían las caricaturas de Teixeira Brandão y Márcio Nery, antiguos directores del HNA, acompañadas de retratos a pluma de internos, que más recuerdan parodias que otra cosa. Se trataba de crónica ilustrada que denunciaba la situación de precariedad de las instalaciones y de los procedimientos de internación en el HNA. Por su parte, la primera foto -del Dr. Afranio Peixoto, exdirector del HNA- es de 1909, en un amplio reportaje sobre la Exposición Internacional de Higiene celebrada aquel año y cuya sede fueron los pabellones de la Exposición de 1908, próximos al Hospicio (Almeida, 2006, p.745). En el Correio da Manhã no encontramos ningún reportaje ilustrado con dibujos, apenas poquísimas noticias con fotos, y casi todas referentes a casos de accesos de locura en el entorno de la ciudad.

La sátira también estaba presente en algunas pequeñas notas o reportajes más extensos de $O$ Paiz. Por ejemplo, la nota satírica relativa a la fiesta de carnaval del rey momo, en 1903, cuando diversos internos aprovecharon la circunstancia para protagonizar una fuga del HNA; o el artículo crítico sobre las lote- 
rías, en el que el estado mental de un interno del HNA se aprovecha como motivo irónico ${ }^{15}$. Recurso habitual de la prensa de Río de Janeiro (Lustosa, 1989b), los textos jocosos provocaban la risa con intención de castigar las costumbres. Más que incitar a la desobediencia o la subversión, forzaban a los lectores a pensar sobre ellas, tratando de demonstrar lo subvertidas que se hallaban por aquel entonces.

La variedad de noticias difundidas por la prensa, como medio de comunicación hegemónico en la época, se reflejaba también en los distintos tipos de artículos que mencionaban al Hospicio Nacional de Alienados. En ellos, se informaba acerca de la administración y funcionamiento de la institución, sobre todo en las notas a respeto de los actos del poder público, como ya se ha mencionado, aunque también se publicasen reportajes más extensos que divulgaban los méritos y la problemática virada de la institución. Asimismo, se recogían noticias sobre visitas al Hospicio de personajes ilustres de los mundos de la política y de la ciencia, incluyendo invitados extranjeros ${ }^{16}$; así como notas de divulgación de actividades clínicas, científicas y sociales de la institución y de sus médi$\cos ^{17}$. Otros artículos aludían a internos que, a causa de algún acontecimiento "fuga, episodio de violencia, suicidio, habeas corpus", se convertían en noticia ${ }^{18}$.

EI HNA aparece también en noticias relativas a acontecimientos turbulentos protagonizados por habitantes de la ciudad, cuya solución pasaba por internar a sus protagonistas en el Hospicio. Se incluyen aquí tanto las notas que relatan brevemente que algún miembro de las fuerzas armadas es enviado al HNA, como también reportajes extensos sobre casos en los que la locura aparece como causa o explicación del suceso, y en los que se informa de la reclusión de alguno de los sujetos implicados en el manicomio, como se verá más adelante.

\section{EL HOSPICIO NACIONAL IMPRESO EN LOS DIARIOS: LUGAR DE ASISTENCIA Y LUGAR PARA LOCURA}

Ese conjunto variado de artículos periodísticos y pequeñas notas sobre el HNA, publicados en ambos diarios, presenta distintas ideas sobre la institución que podemos agrupar según tres puntos de vista: los dos primeros se centran en la descripción y el retrato de la vida institucional que la prensa llevó a cabo en sus diferentes aspectos (administrativo, asistencial y científico); mientras que el tercero analiza aquellas situaciones en que el Hospicio se contempla como una solución para los acontecimientos que perturbaban la vida de la ciudad.
El primer punto de vista concentra artículos en los que el HNA se observa desde una visión positiva, como lugar dedicado a la gestión y al tratamiento adecuado de la enfermedad mental, y también como una suerte de escaparate del desarrollo científico de la nación. Son varias las notas y artículos que informan sobre el buen funcionamiento de la institución: rendición de cuentas, concursos y nombramientos de nuevos médicos y funcionarios, obras realizadas, inauguraciones, etc. Esta visión positiva sobre el Hospicio también aparece en artículos acerca de las personalidades científicas que lo visitaban. La institución era el "ojito derecho" de la Asistencia a Alienados y puerta de entrada a sus establecimientos públicos en el ámbito de la Capital Federal:

[...] El Hospicio, como se sabe, honra a nuestra Administración Pública; sus diversos servicios presentan toda la pulcritud y los cuidados que se pueda desear. Los congresistas extranjeros se han mostrado encantados con el buen orden que allí encontraron, elogiando sobremanera el estado de limpieza y los modernos aparatos científicos de los distintos pabellones. [...]. ${ }^{19}$

Esas noticias, difundidas en ambos periódicos, pero de modo más recurrente en $O$ Paiz, muestran una percepción muy elogiosa del Hospicio Nacional, al que consideraban modelo de lo que existía de más moderno y científico en la joven República, sin que nada tuviera que envidiar a las naciones extranjeras. Además, se reproducían también notas frecuentes sobre los médicos que a menudo eran invitados a participar en congresos o eventos internacionales, principalmente Juliano Moreira ${ }^{20}$, lo que confirmaba una vez más la divulgación de la relevancia de la institución.

El segundo punto de vista presenta los límites e irregularidades en las reformas planeadas, tanto las financiero-administrativas como aquellas situaciones que ponían en riesgo la vida y la integridad de los internos, así por ejemplo, las provocadas por las pésimas condiciones sanitarias, por los imponderables de la locura o por el uso de la violencia institucional. Sobre este aspecto, encontramos artículos en los que se relaciona a la institución con ciertos escándalos que los diarios presentaban en detalle ante la opinión pública, como la falta de pago de los sueldos de empleados ${ }^{21}$ o los problemas de suministro de productos para la institución ${ }^{22}$, entre otros.

En esta línea, un caso que ocupó las páginas del Correio da Manhã el 23 de enero de 1904 fue el de una carta de reclamación dirigida al diario por un médico del Hospicio Nacional no identificado. Tal carta denunciaba una serie de nombramientos im- 
procedentes en la institución que supondrían una violación del reglamento vigente, el cual garantizaba la realización de un concurso público. El tono de denuncia respecto a las arbitrariedades del poder público está en conexión con la línea editorial del Correio da Manhã:

[...] Como bien saben, deberá entrar en vigor estos días el reglamento interno del Hospicio Nacional de Alienados. [...] Con tal motivo, se efectuarán los nombramientos para los cargos previstos en él. Sucede, sin embargo, que estos nombramientos, correspondientes al inicio de la ejecución del reglamento, suponen su violación de todo punto. [...] Ha sido una disposición siempre aceptada la de que, para los cargos de médico, se prefiera a los que hayan trabajado como residentes. Sin embargo, se va a consumar el atentado de nombrar como médico adjunto a quien es absolutamente desconocedor de la materia, poquísimas veces pisó sus aulas y jamás disfrutó de las lecturas de psiquiatría. [...] La ley establece las oposiciones y el Gobierno debe cumplirlas en todos sus puntos. Que el Correio da Manhã, que ha sido siempre el defensor de las causas nobles, no abandone a aquellos que solo confían en su trabajo y esfuerzo propios. ${ }^{23}$

El fragmento presentado apunta las arbitrariedades del Estado y del Hospicio Nacional en el proceso de nombramiento de médicos para dicha institución, contrariando las directrices establecidas por la ley. Refleja, así, el papel de la prensa como agente político, no solo al denunciar actos ilegales cometidos por el propio Estado (o al contrario, por alabar las acciones del Gobierno y del Hospicio), sino también, en este caso concreto, al garantizar al ciudadano el derecho a la palabra.

La idea de una institución poco eficaz, e incluso desvirtuada, aparece también en el relato de casos que retrataban el lado sombrío de la vida en el interior de la institución: violaciones, violencia, muerte, suicidio... A veces, un mismo caso era asunto para varios artículos en ambos diarios, como ocurrió con la violación de una interna del Hospicio a manos de un pintor que trabajaba en las obras del HNA en 1904. En relación con este suceso, "se contaba la misma historia" en Correio da Manhã y en O Paiz, si bien en un tono mucho más complaciente y con ciertas matizaciones en el último ${ }^{24}$ (Silva, 2014, pp. 201-203).

Otros muchos casos de violencia en la institución aparecen en las páginas de ambos diarios, como la muerte de un alienado por los malos tratos de un enfermero del Hospicio Nacional. El Correio da Manhã, del 07 de marzo de 1908 lo refería de esta forma:
Un caso muy grave es el de un pobre enfermo, allí ingresado, que ha muerto a consecuencia de los malos tratos a los que se ha visto sometido por uno de los funcionarios encargado de vigilarlo y que era responsable de su vida. No hay palabras para justificar tan monstruosa irregularidad en el servicio de aquella casa. $[\ldots . .]^{25}$

Menos comunes, aunque con cierta incidencia, son los casos noticiados en que los internos piden la realización de nuevos exámenes sanitarios, alegando haber sido ingresados en el Hospicio sin que sufrieran enfermedad mental alguna. El 28 de mayo de 1903, por ejemplo, O Paiz publica un artículo sobre un expediente abierto por un interno que afirmaba haber sido ingresado en el Hospicio Nacional sin estar loco, motivo por el que se determinó la realización de una nueva prueba médica. A veces, estas noticias recogían las manifestaciones de los propios internos, como el caso que ocupó las páginas del Correio da Manhã el 11 de junio de 1907, cuyo enredo había comenzado en 1901. Se trata de una denuncia por maltrato y violación de las garantías individuales presentada por un exinterno del Hospicio Nacional, por medio de las cartas publicadas en tres ocasiones en el periódico citado:

"Hago público el hecho de que, encerrado en el Hospicio Nacional de Alienados el 18 de enero de 1901, por un incalificable abuso del Gobierno de entonces y de sus agentes, ya que no presentaba ninguna alteración de mis facultades mentales, conforme he afirmado en anteriores instancias y declaraciones al Gobierno [...], fui víctima en el citado establecimiento de los mayores martirios y vejaciones. [...] fui llevado por mis agresores, a quienes nada había hecho, a las dependencias del primer piso de la parte delantera del edificio, para ser allí maltratado de distintas maneras. [...] Mi objetivo con esta publicación es declarar púbicamente al Gobierno como responsable de esos crímenes y de las consecuencias que de ellos puedan surgir, pues quienes colaboraron de algún modo en mi arresto solo deseaban inutilizarme por medio de la muerte o de la locura, contando para ello con el servilismo y cobardía de los directores del Hospicio Nacional y de sus auxiliares". ${ }^{26}$

Las noticias relacionadas con el tercer punto de vista presentan como principal característica la percepción del Hospicio Nacional de Alienados como un recinto reservado de forma apropiada para la locura, adonde se envían los individuos que se suponía podían presentar alienación mental o conductas desviadas ligadas a ella. Se difundieron en este período dos artículos sobre la transferencia de internos del Asilo São Francisco de Assis al manicomio (1907 y 1908), así como otras muchas notas, a menudo en la columna "Ejército", sobre el envío de miembros de las fuerzas armadas a la ins- 
titución. Se trataba de marineros y soldados -aunque también oficiales del ejército, un teniente, un capitán teniente y un mayor- que aparecen mencionados en las páginas de los periódicos por su internación, baja, alta o, incluso, denuncia de los trámites institucionales del manicomio (este último caso publicado únicamente en el Correio da Manhã). Por otro lado, a partir de 1911 comienzan a aparecer con frecuencia en la "Sección Policial" de $O$ Paiz notas sobre indigentes recogidos por la policía y llevados al HNA ${ }^{27}$.

Este tercer punto de vista también se hace presente en varios artículos que informan sobre casos habituales de individuos que, tras ser detenidos por la policía, son enviados al Hospicio Nacional por su participación en un hecho considerado como escandaloso en la vida de la ciudad ${ }^{28}$. Los implicados son personas identificadas como exinternos de la institución o bien nunca antes internadas en ella. En los dos periódicos, la información sobre esos acontecimientos o incidentes se publicaban en notas en columnas -como "En la policía y en las calles", del Correio da Manhã-, y también en artículos cortos, con títulos específicos, o en otros más extensos con destaque gráfico en el título y en el empleo de fotos, de las pocas publicadas en este período, como ya se ha señalado. Así, O Paiz y el Correio da Manhã presentan el manicomio como el lugar apropiado para acoger a individuos identificados según diferentes categorías: tentativa de suicidio, suicidio consumado, crimen (asesinato, decapitación), locura peligrosa, acceso de locura, ataque de locura, ataque de alienación, sospecha de alienación, borrachera, agresión y botellazo, manía persecutoria...; o por ser loca, alucinada, alienado, maníaco, epiléptico o loco (loco infeliz, loco furioso, pobre loco, loco que se cree Cristo). Tales artículos reseñaban el acontecimiento desde el punto de vista de quien "estuvo allí" o de quien "escuchó a alguien que estuvo allí".

Entre los muchos casos que podríamos citar está el del suicida maníaco, publicado en forma de pequeño texto en el Correio da Manhã, el 18 de noviembre de 1903:

Es incorregible este francés Joannes Aturant, que hace tiempo decidió suicidarse, suministrando así noticias a los periódicos con sus constantes tentativas contra la propia existencia. Hace pocos días, el 14 del corriente, Joannes intentó suicidarse [...]. Ayer, apenas tres días después, volvió el hombre a atentar contra su vida. En la misma casa, en el número 21 de la calle Senador Dantas, ingiriendo la misma dosis del mismísimo tóxico, trató de llevar a cabo su lúgubre y triste ocurrencia. A continuación, tomó un tílburi, ordenando al cochero que se dirigiera a la Comisaría Central de la Policía [...]. Como el hecho había ocurrido en la calle Senador Dantas, le dijeron al suicida incorregible que se presentase ante las autoridades de la 7a [comisaría de policía], encaminándose el vehículo para dicha comisaría. El comisario, por segunda vez, lo mandó al hospital de la Misericordia. Mejor sería que la autoridad enviase a Joannes al Hospicio de Alienados... ${ }^{29}$

Este también fue el caso relatado por O Paiz el 19 de octubre de 1909 con el título de "Locura Peligrosa":

\begin{abstract}
Vivían en el mismo aposento de la casa de dos cuartos de la calle del Espírito Santo n.o 39 dos hermanos, Luiz Fernandes da Costa y Antonio da Costa, ambos trabajadores. En la madrugada de ayer, Luiz, acometido de un acceso de locura, se levantó, se dirigió al patio de la casa y se hizo allí con un hacha para asesinar a su hermano. Antonio dormía y se habría convertido en víctima fatal de no ser porque el golpe dirigido contra él por su hermano no llegó a acertarle [...]. El pobre loco iba a golpearlo de nuevo, cuando Antonio, herido, se levantó de un salto y, percibiendo la situación, se abalanzó sobre Luiz, con quien luchó algunos minutos. Consiguió así desarmarlo. Después, ayudado por algunas personas de la casa que habían acudido al alboroto, amarró a su hermano loco y fue a llamar a la policía. La comisaría del 4 ㅇ Distrito determinó que Luiz Fernandes fuese trasladado en furgón policial a la sede central de la policía, de donde partió a primeras horas del día para el pabellón de observación del Hospicio de Alienados. ${ }^{30}$
\end{abstract}

Estos casos violentos y escandalosos dicen mucho, considerando las categorías ya citadas, sobre las representaciones en torno a la locura y los locos publicadas en los diarios O Paiz y Correio da Manhã. Se trata de una etiqueta social que abarca a individuos que ya cargaban consigo esa identidad, marcada por ser exinterno del HNA. Otros son identificados públicamente como locos por primera vez, aunque en el momento de la divulgación del caso por los diarios, sus familiares y otras personas recuerden indicios y hechos pasados que ya revelarían su condición de locura. Podían ser violentos e incluso criminales, y otras veces dignos de pena y benevolencia como el pobre loco. Son vistos también como enfermos, según expresan las categorías de alienado, epiléptico, maníaco. Además, estos artículos periodísticos muestran cómo existía un modus operandi socialmente instituido para tratar la locura. En ellos se relataban y difundían los procedimientos considerados correctos en tales casos: los habitantes de la ciudad debían defenderse ante cualquier situación de violencia y llamar a la policía cuando consideraran que estaba teniendo lugar un acceso, un ataque o incluso una sospecha de alienación; todo ello a pesar de que la distinción o asociación de la locura con la violencia, contra uno o contra el prójimo, fuese, a veces, nebulosa. 
El análisis más detenido y futuro de los diversos casos divulgados en $O$ Paiz y el Correio da Manhã habrá de profundizar en la comprensión de esos procesos de identificación social de la locura que definían a los sujetos susceptibles de ser internados en el Hospicio Nacional de Alienados. No obstante, por el momento, consideramos haber demostrado que las noticias acerca de esta institución, recogidas por la prensa republicana mencionada, abordaban variados asuntos relativos a su presencia en la ciudad de Río de Janeiro, tanto en lo referente a la institución propiamente dicha, a sus médicos, funcionarios y pacientes, como en lo tocante a acontecimientos cuyo desenlace implicaba el envío de algún individuo para ser internado en el manicomio.

Como hilos de conexión entre esta variedad temática periodística se ha podido observar la divulgación simultánea de diferentes representaciones o puntos de vista sobre el HNA. El Hospicio, que había sido creado bajo la promesa de asistencia a los alienados en el período imperial, se reinventa como tal para adecuarse a las nuevas directrices republicanas de asistencia, ciencia y modernidad urbana. De este modo, el manicomio se beneficia de esas directrices en los espacios de su entorno, así como en las reformas de sus instalaciones y procedimientos de tratamiento, apareciendo a ojos de la población como un icono más de la organización de la salud pública y de los avances de la ciencia médica del país. Al mismo

\section{NOTAS}

1. La institución se llamó Hospicio de Pedro II durante el Imperio. En 1890, al año siguiente de la proclamación de la República, pasó a denominarse Hospicio Nacional de Alienados, y en 1911 recibió el nombre de Hospital Nacional de Alienados. En 1927 vuelve a cambiar su denominación por el de Hospital Nacional de Psicópatas.

2. Pedro II era un niño cuando le tocó asumir el trono imperial, motivo por el cual se nombró una regencia para que gobernase en su lugar hasta que alcanzara la mayoría de edad en 1843. Las disputas entre facciones políticas desembocaron en varias revueltas y condujeron a una situación política muy inestable. La posibilidad de anticipar la mayoría de edad para que el joven emperador gobernase ya se consideraba desde 1835 y, en cierto modo, recibía el apoyo de los dos principales partidos políticos. Con la anuencia del joven emperador, la Asamblea General (parlamento brasileño) declaró formalmente a Pedro II mayor a los 14 años en 1840, siendo aclamado, coronado y consagrado el 18 de julio de 1841.

3. La mayor parte de los estudios que analizan la prensa en relación con la historia de la psiquiatría lo hacen tomándola como una tiempo, y con mayor vehemencia en el Correio da Manhã, la prensa analizada divulgaba también una imagen negativa del manicomio, al publicar los límites y males de la institución, no tan eficaz en lo que se refería a su administración y misión terapéutica, si se tienen en cuenta las diversas noticias sobre abusos y violencia que ocurrían en el interior del establecimiento. Con todo, no por eso el Hospicio perdía su valor dentro de la ciudad. Además de las representaciones positivas o negativas para el prestigio del Hospicio Nacional, la prensa ayudaba a mantener el lugar social de esta institución al informar y opinar sobre quién era enviado al manicomio y de qué modo esto debía ocurrir.

\section{AGRADECIMIENTOS}

Investigación financiada por el CNPq a través de una beca del Programa de Iniciación Científica CNPq/ Fiocruz, concedida a José Roberto Saiol (2015), y con el apoyo de la Casa de Oswaldo Cruz/Fiocruz a través de una beca para Caroline Gonzaga de Oliveira (2016), estudiante de la carrera de Historia de la Universidad del Estado de Río de Janeiro. Agradecemos a Ana Lucia Vieira dos Santos (Escuela de Arquitectura y Urbanismo del Universidad Federal Fluminense) por la preciosa ayuda con la investigación cartográfica y preparación gráfica del mapa; a Maria Belén Posada Alonso y a Bethania Guerra de Lemos por la colaboración lingüística y la versión en español del artículo.

fuente a ser cotejada con otro tipo de documentos. Otros enfoques, menos frecuentes, abordan la prensa como fuente y objeto de estudio para la historia de la psiquiatría a la vez (Ayala Flores, 2004; Sacristán, 2009, pp. 164 y 166). Adoptamos aquí esta última perspectiva, al considerar las noticias en la prensa como una serie documental que, analizada en su conjunto y en su organicidad, es agente principal para la producción del imaginario social sobre el Hospicio que circuló en el inicio de la Primera República brasileña.

4. Fuentes disponibles en la Hemeroteca Digital de la Biblioteca Nacional de Brasil, sobre las que se ha investigado con las siguientes palabras clave: Hospicio de Alienados; Hospicio Nacional, Hospital Nacional.

5. Otros periódicos representativos de la prensa profesional fueron el Jornal do Commercio (1824) y el Jornal do Brasil (1891) (Tavares, 1980, p. 30). A buen seguro el desarrollo de futuras investigaciones sobre las noticias a respecto del Hospicio en esos otros diarios podrá dar lugar a generalizaciones más amplias de los resultados aquí divulgados, así como corroborar los pormenores de las noticias que sobre el Hospicio se publicaron en O Paiz y en el Correio da Manhã. 
6. O Paiz, 25 y 28 de enero de 1902; Correio da Manhã, 24, 25, 26 y 28 de enero de 1902 y 06.02.1902. Véase Azevedo (2012).

7. La historiografía más reciente sobre el proceso político durante la Primera República, señala que la "política de los gobernadores" no eliminó por completo los conflictos locales, así como no presentaba garantía absoluta de hegemonía, libre de conflictos, para los estados de Minas y São Paulo (Viscardi, 2001; Resende 2008). La rotación entre estos dos estados de la federación en las sucesiones presidenciales implicaba negociaciones complejas en las que también se tenían en cuenta los intereses de los estados menos representados en el Congreso (Resende, 2008, 118).

8. Bocaiúva había sido el primer ministro de las Asuntos Exteriores (1889-1891) durante el gobierno del mariscal Hermes da Fonseca.

9. Leal $(s / d), s / p$.

10. Arquivo Nacional do Rio de Janeiro, Fundo/coleção Relação do Rio de Janeiro - 84. Ouvidoria Geral. Susano, Manuel Antunes (Inventariado), Inventario 1783, no 22, caja 3629.

11. Aunque el área entorno al Hospicio sufrió transformaciones, no fue objeto de inversión ni tampoco apreciada como zona residencial o comercial, sobre todo, en comparación con la zona central de la ciudad y otros lugares a la orilla del mar. Los cambios en el diseño urbanístico de la Praia da Saudade se materializaron apenas a partir de 1920, con las reformas implantadas por el alcalde Carlos Sampaio (1920-1922) (Abreu, 2013, pp. 76-77).

12. O Paiz, 02.04.1903, 09.06.1903, 13.09.1903 y 29.03.1904 .

13.O Paiz, 1.07.1905 y 13.07.1905.

14. Son muchos los registros, pudiéndose citar como ejemplos: O Paiz, 09.03.1904 (nombramiento), 18.08.1904 (rendición de cuentas), 24.08.1907 (licencia de funcionario), 10.04.1908 (concurso público/oposiciones) y Correio da Manhã, 30.01.1910 (autorización del ministro), 23.03.1910 (concurso público/oposiciones).

15. Respectivamente, O Paiz, 16.02.1903; 23.12.1908.

16. A modo de ejemplo, véase $O$ Paiz, 06.01.1905 y Correio da Manhã, 22.10.1909, sobre la visita del ministro del Interior; y $O$ Paiz, 28.07.1907, sobre la visita del presidente de la República.
17. Pudiéndose citar como ejemplos: O Paiz, 10.01.1904, acerca del nombramiento de Juliano Moreira como miembro del consejo editorial del periódico Brazil Médico; O Paiz, 22.08.1904, nota sobre el cumpleaños del ministro J.J. Seabra, al que asistió Juliano Moreira; O Paiz, 13.06.1907, felicitaciones por el cumpleaños de Fernandes Figueira, pediatra del HNA; O Paiz, 29.05.1908, nota sobre la publicación de un libro científico que divulga investigaciones realizadas en el HNA, y el Correio da Manhã, 18.09.1907 y 12.03.1910.

18. Ejemplos: O Paiz, 28.05.1903, sobre una solicitud de habeas corpus; O Paiz, 08.02.1904, sobre una fuga del HNA; y O Paiz, 09.05.1908, sobre violencia ejercida a un interno del Hospicio.

19. O Paiz, "Exposição de Higiene", 03.08.1909, p.3. Noticia sobre la visita al Hospicio Nacional de delegados extranjeros del Congreso Médico, con ocasión de la Exposición de Higiene.

20.A modo de ejemplo, véase Correio da Manhã, 16.04.1909.

21.O Paiz, 08.03.1904; Correio da Manhã, 13.05 .1910 y 22.09.1910.

22. O Paiz 16, 17 y 30 de julio de 1903.

23. Correio da Manhã,,"Hospício de Alienados", 23.01.1904, p.1.

24. O Paiz , “O caso do Hospício”, 21.05.1904 y Correio da Manhã, "Um caso escandaloso no Hospício Nacional", 21.05.1904.

25. Correio da Manhã, "Um crime no hospício", 07.03.1908, p.8.

26. Correio da Manhã, "Hospício Nacional", 11.06.1907, p.6.

27. O Paiz, 24.02.1911 y 11.03.1911.

28. Algunos ejemplos pueden encontrarse en $O$ Paiz, 23.03.1901; 18 .09.1902; 11.12 .1903 y en el Correio da Manhã, 20.06.1903; 18.11.1906 y 21.07.1910.

29. Correio da Manhã , "Suicidomaníaco", 18.10.1903, p.2.

30. O Paiz, "Locura Peligrosa", 19.10.1909, p.7. 


\section{BIBLIOGRAFÍA}

Ablard, Jonathan D. (2008), Madness in Buenos Aires. Patients, Psychiatrists and the Argentine State (1880-1983), Canada, University of Calgary Press.

Abreu, Mauricio de Almeida (2013), A Evolução urbana do Rio de Janeiro, 4a ed., Rio de Janeiro, Instituto Pereira Passos.

Almeida, Marta de (2006), "Circuito aberto: ideias e intercâmbios médico-científicos na América Latina nos primórdios do século XX", História, Ciências, Saúde - Manguinhos, 13 (3), pp. 733-757.

Alonso, Angela (2002), Idéias em Movimento: a geração de 1870 na crise do Brasil-Império, São Paulo, Paz \& Terra.

Ayala Flores, Huborno (2004), "Los dementes, la locura y el manicomio a través de los periódicos El Reproductor y El Cosmopolita de Orizaba: 1876-1911". En: Bicentenario de la prensa provincial en México. Estudios sobre periodismo veracruzano, Veracruz, Instituto Veracruzano de la Cultura, pp. 45-65.

Azevedo, Roberto Cesar Silva de (2012), O desfalque, o inquérito e as comissões de inspeção: a assistência a alienados no Brasil (1902-1925), (Dissertação de Mestrado), Rio de Janeiro, Departamento de História da Pontifícia Universidade Católica.

Campos Marín, Ricardo y Huertas, Rafael (2008), “Los lugares de la locura: reflexiones historiográficas en torno a los manicomios y su papel en la génesis y el desarrollo de la psiquiatría", Arbor: ciencia, pensamiento y cultura, CLXXXIV, pp. 471-480.

Carvalho, Maria Célia Freire de (1980), “Estudo de caso: Correio da Manhã". En: Alves, Ivan y Tavares, José Nilo (eds.), A imprensa na década de 20. (datilog.). Rio de Janeiro, Associação Brasileira de Imprensa, FINEP, pp. 96-108.

Engel, Magali [1995] (2001), Os delírios da razão: médicos, loucos e hospícios, Rio de Janeiro, 1830-1930, Rio de Janeiro, Fiocruz.

Facchinetti, Cristiana; Chagas, Daiana Cruz; Ribeiro, Andrea y Reis, Cristiane Sá. (2010), "No labirinto das fontes do Hospício Nacional de Alienados", História, Ciências, Saúde - Manguinhos, 17 (supl.2), pp. 733-768.

Fausto, Boris (2002), História concisa do Brasil, São Paulo, Edusp y Imprensa Oficial do Estado.

Gonçalves, Monique de Siqueira; Edler, Flavio Coelho (2009), "Os caminhos da loucura na Corte Imperial: um embate historiográfico acerca do funcionamento do Hospício Pedro II de 1850 a 1889", Revista Latinoamericana de Psicopatologia Fundamental, 12 (2), pp. 393-410.

Gonçalves, Monique de Siqueira (2013), “Os primórdios da Psiquiatria no Brasil: o Hospicio Pedro II, as casas de saúde particulares e seus pressupostos epistemológicos (1850-1880)", Revista Brasileira de História da Ciência, 6 (1), pp. 60-77.
Huertas, Rafael (2009), “Medicina social, control social y políticas del cuerpo. La Subjetivación de la norma". En: Miranda, Marisa y Girón Sierra, Álvaro (coords.) Cuerpo, biopolítica y control social: América Latina y Europa en los siglos XIX y XX, Buenos Aires, Siglo XX.

Huertas, Rafael (2012), Historia cultural de la psiquiatria: (re) pensar a locura, Madrid, Catarata.

Leal, Carlos Eduardo (s/d), “O Paiz”, Dicionário Histórico-Biográfico Brasileiro, Rio de Janeiro, Centro de Pesquisa e Documentação de História Contemporânea do Brasil - Fundação Getúlio Vargas (CPDOC-FGV). Disponible en: http://www. fgv.br/cpdoc/acervo/dicionarios/verbete-tematico/pais-o [consultado el 3/01/2017].

Lustosa, Isabel (1989a), Histórias de Presidentes: a República no Catete, Petrópolis- Rio de Janeiro, Ed Vozes-Fundação Casa de Rui Barbosa.

Lustosa, Isabel (1989b), “Humor e política na Primeira República”, Revista USP, 53, pp. 53-64.

Machado, Roberto; Loureiro, Angela; Luz, Rogerio; Muricy, Katia (1978), Danação da Norma: a medicina social e constituição da psiquiatria no Brasil, Rio de Janeiro, Graal.

Martins, Ana Luiza; Luca, Tania Regina de (2006), Imprensa e cidade, São Paulo, UNESP.

Melo, Victor Andrade de (2001), "Os clubes de remo e as relações com as autoridades governamentais". En: Cidade sportiva: primórdios do esporte no Rio de Janeiro, Rio de Janeiro, Relume-Dumará, pp.93-106.

Munõz, Pedro; Facchinetti, Cristiana; Dias, Allister Teixeira (2011), "Suspeitos em observação nas redes da psiquiatria: o Pavilhão de Observações (1894-1930)", Memorandum, 20 (abr.), Belo Horizonte, UFMG, Ribeirão Preto, USP.

Nascimento, Maria Isabel Moura; Zanlorenzi, Claudia Maria Petchak (2006), "Imprensa no Brasil: do Império à Primeira República", Acervo, Rio de Janeiro, 19 (12), pp. 37-52.

Pereira, Margareth da Silva (2010), “A Exposição de 1908 ou o Brasil visto por dentro", Arqtexto, 16, pp. 6-27.

Resende, Maria Efigênia Lage de (2008), “O processo político na Primeira República e o liberalismo oligárquico”. En: Ferreira, Jorge y Delgado, Lucilia de Almeida Neves (orgs.), O Brasil republicano v.1 - O tempo do liberalismo excludente: da Proclamação da República à Revolução de 1930, Rio de Janeiro, Civilização Brasileira, pp. 89-120.

Ribeiro, Daniele Corrêa (2015), Tramas da loucura na Corte Imperial: ciência, caridade e redes de sociabilidade no Hospício de Pedro II (1883-1889), Curitiba, Prismas.

Ribeiro, Daniele Corrêa (2016), Os sentidos do Hospício de Pedro Il: dinâmicas sociais na constituição da psiquiatria brasi- 
leira (1841-1889), (Tese de Doutorado), Rio de Janeiro, Casa de Oswaldo Cruz, Fiocruz.

Ríos Molina, Andrés (2009), La locura durante la Revolución Mexicana: los primeros años del Manicomio General La Castañeda, 1910-1920, México, D.F, Colegio de México.

Sacristán, Cristina (2005), "Historiografía de la locura y de la psiquiatría en México. De la hagiografía a la historia posmoderna", Frenia. Revista de Historia de la Psiquiatría, 5 (1), pp. 9-33.

Sacristán, Cristina (2009), "La locura se topa con el manicomio: Una historia por contar", Cuicuilco, 16 (45), pp. 163-189.

Silva, Renata Prudencio da (2014), As ciências de Afranio Peixoto: higiene, psiquiatria e medicina legal (1892-1935), (Tese de Doutorado), Rio de Janeiro, Casa de Oswaldo Cruz, Fiocruz.

Sodré, Nelson Werneck (1983), História da imprensa no Brasil (3a. ed.), São Paulo, Martins Fontes.

Souza, Maria do Carmo Campello de (1968), “O processo político partidário na Primeira República". En: Cardoso, Fernando
Henrique (org.) Corpo e alma do Brasil, vol. XXIII, São Paulo, Difusão Européia do Livro.

Tavares, José Nilo (1980), "Visão geral da sociedade e da imprensa na década de 20". En: Alves, Ivan; Tavares, José Nilo (eds.) A imprensa na década de 20, Rio de Janeiro, Associação Brasileira de Imprensa, FINEP, pp. 4-33

Teixeira, Manoel Olavo (1998), Deus e a ciência na terra do sol: o Hospiciode Pedro II e a constituição da medicina mental no Brasil, (Tese de Doutorado), Rio de Janeiro, Instituto de Psiquiatria da Universidade Federal do Rio de Janeiro.

Venancio, Ana Teresa A. y Carvalhal, Lázara de Azevedo (2005), "Juliano Moreira: a psiquiatria científica no processo civilizador brasileiro". En: Duarte, Luiz Fernando Dias; Russo, Jane y Venancio, Ana Teresa (orgs.). Psicologização no Brasil: atores e autores, Rio de Janeiro, Contra Capa, pp. 65-83.

Viscardi, Cláudia Maria Ribeiro (2001), O Teatro das oligarquias: uma revisão da "política do café com leite", Belo Horizonte, C/Arte. 